\title{
Neutrino properties from high energy astrophysical neutrinos
}

\author{
Sandip Pakvasa ${ }^{a}$ \\ a Department of Physics and Astronomy, University of Hawaii, Honolulu, HI 96822, USA \\ It is shown how high energy neutrino beams from very distant sources can be utilized to learn about some \\ properties of neutrinos such as lifetimes, mass hierarchy, etc. Furthermore, even mixing elements such as $U_{e 3}$ and \\ the CPV phase in the neutrino mixing matrix can be measured in principle. Pseudo-Dirac mass differences as \\ small as $10^{-18} e V^{2}$ can be probed as well.
}

\section{Introduction}

We make two basic assumptions which are reasonable. The first one is that distant neutrino sources (e.g. AGN's and GRB's) exist; and furthermore with detectable fluxes at high energies (upto and beyond $\mathrm{PeV}$ ). The second one is that in the not too far future, very large volume, well instrumented detectors of sizes of order of KM3 and beyond will exist and be operating; and furthermore will have (a) reasonably good energy resolution and $(\mathrm{b})$ good angular resolution $\left(\sim 1^{0}\right.$ for muons).

\section{Neutrinos from Astrophysical Sources}

If these two assumptions are valid, then there are a number of uses these detectors can be put to 1. In this talk I want to focus on those that enable us to determine some properties of neutrinos: namely, probe neutrino lifetimes to $10^{4} \mathrm{~s} / \mathrm{eV}$ (an improvement of $10^{8}$ over current bounds), pseudo-Dirac mass splittings to a level of $10^{-18} \mathrm{eV}^{2}$ (an improvement of a factor of $10^{6}$ over current bounds) and potentially even measure quantities such as $U_{e 3}$ and the phase $\delta$ in the MNSP matrix [2].

\section{Astrophysical neutrino flavor content}

In the absence of neutrino oscillations we expect a very small $\nu_{\tau}$ component in neutrinos from astrophysical sources. From the most discussed and the most likely astrophysical high energy neutrino sources [3] we expect nearly equal numbers of particles and anti-particles, half as many $\nu_{e}^{\prime} s$ as $\nu_{\mu}^{\prime} s$ and virtually no $\nu_{\tau}^{\prime} s$. This comes about simply because the neutrinos are thought to originate in decays of pions (and kaons) and subsequent decays of muons. Most astrophysical targets are fairly tenous even compared to the Earth's atmosphere, and would allow for full muon decay in flight. There are some predictions for flavor independent fluxes from cosmic defects and exotic objects such as evaporating black holes. Observation of tau neutrinos from these would have great importance. A conservative estimate 4] shows that the prompt $\nu_{\tau}$ flux is very small and the emitted flux is close to the ratio $1: 2: 0$. The flux ratio of $\nu_{e}: \nu_{\mu}: \nu_{\tau}=1: 2: 0$ is certainly valid for those AGN models in which the neutrinos are produced in beam dumps of photons or protons on matter, in which mostly pion and kaon decay (followed by the decay of muons) supply the bulk of the neutrino flux.

Depending on the amount of prompt $\nu$-flux 
due to the production and decay of heavy flavors, there could be a small non-zero $\nu_{\tau}$ component present. There are also possible scenarios in which the muons lose energy in matter or in strong magnetic fields [5], in which case the initial flux mixture becomes $\nu_{e}: \nu_{\mu}: \nu_{\tau}=0: 1: 0$.

\section{Effect of Oscillations}

The current knowledge of neutrino masses and mixings can be summarized as follows [6]. The mixing matrix is given to a good approximation by

$U=\left(\begin{array}{ccc}c & s & \epsilon \\ s / \sqrt{2} & c / \sqrt{2} & 1 / \sqrt{2} \\ s / \sqrt{2} & c / \sqrt{2} & 1 / \sqrt{2}\end{array}\right)$

where $c=\cos \theta, s=\sin \theta$ with $\theta$ the solar mixing angle given by about $32^{0}$ and $\epsilon=U_{e 3}<0.17 \mathrm{lim}$ ited by the CHOOZ bound. The mass spectrum has two possibilities; normal or inverted, and with the mass differences given by $\delta m_{32}^{2} \sim 2.10^{-3} \mathrm{eV}^{2}$ and $\delta m_{21}^{2} \sim 7.10^{-5} e V^{2}$. Since $\delta m^{2} L / 4 E$ for the distances to GRB's and AGN's (even for energies upto and beyond $\mathrm{PeV})$ is very large $\left(>10^{7}\right)$ the oscillations have always averaged out and the conversion and survival probabilities are given by

$$
\begin{aligned}
P_{\alpha \beta} & =\sum_{i}\left|U_{\alpha i}\right|^{2}\left|U_{\beta i}\right|^{2} \\
P_{\alpha \alpha} & =\sum_{i}\left|U_{\alpha i}\right|^{4}
\end{aligned}
$$

Assuming no significant matter effects enroute, the mixing matrix in Eq. (1) leads to a propagation matrix $\mathrm{P}$, given by:

$P=\left(\begin{array}{ccc}1-S / 2 & S / 4 & S / 4 \\ S / 4 & 1 / 2-S / 8 & 1 / 2-S / 8 \\ S / 4 & 1 / 2-S / 8 & 1 / 2-S / 8\end{array}\right)$

where $\mathrm{S}$ stands for $\sin ^{2}(2 \theta)$. As is obvious, for any value of the solar mixing angle, $\mathrm{P}$ converts a flux ratio of $\nu_{e}: \nu_{\mu}: \nu_{\tau}=1: 2: 0$ into one of
$1: 1: 1$. Hence the flavor mix expected at arrival is simply an equal mixture of $\nu_{e}, \nu_{\mu}$ and $\nu_{\tau}$ as was observed long ago 47. An initial flavor mix of $\nu_{e}: \nu_{\mu}: \nu_{\tau}=0: 1: 0$ is converted by oscillations into one of about $1 / 2: 1: 1$. There are several other ways, arising from intrinsic properties of neutrinos, by which the flavor mix can change from the canonical $1: 1: 1$ figure. One in particular, which gives rise to striking signatures, is the decay of neutrinos $[8]$. Before discussing the other possibilities, let me consider the case of neutrino decay.

\section{Neutrino Decay 9}

We now know that neutrinos have non-zero masses and non-trivial mixings, based on the evidence for neutrino mixings and oscillations from the data on atmospheric, solar and reactor neutrinos.

If this is true, then in general, the heavier neutrinos are expected to decay into the lighter ones via flavor changing processes. The only questions are (a) whether the lifetimes are short enough to be phenomenologically interesting (or are they too long?) and (b) what are the dominant decay modes.

Throughout the following, to be specific, I will assume that the neutrino masses are at most of order of eV. Since we are interested in decay modes which are likely to have rates (or lead to lifetimes) which are phenomenologically interesting, we can rule out several classes of decay nodes.

First, consider radiative decays, such as $\nu_{i} \rightarrow$ $\nu_{j}+\gamma$. Since the experimental bounds on $\mu_{\nu_{i}}$, the magnetic moments of neutrinos, come from reactions such as $\nu_{e} e \rightarrow e^{\text {" }} \nu$ " which are not sensitive to the final state neutrinos; the bounds apply to both diagonal as well as transition magnetic mo- 
ments and so can be used to limit the corresponding lifetimes. The bounds should really be on mass eigenstates [10, but since the mixing angles are large, it does not matter much. The current bounds are[11]:

$\tau_{\nu_{e}}>5.10^{18} \mathrm{sec}$

$\tau_{\nu_{\mu}}>5.10^{16} \mathrm{sec}$

$\tau_{\nu_{\tau}}>2.10^{11} \mathrm{sec}$

In the above limits the first one gives a bound for the $\tau_{\nu_{1}}$, whereas the second one gives the bound for both $\tau_{\nu_{2}}$ as well as $\tau_{\nu_{3}}$ since the mixing is essentially maximal.

There is one caveat in deducing these bounds. Namely, the form factors are evaluated at $q^{2} \sim$ $O\left(e V^{2}\right)$ in the decay matrix elements whereas in the scattering from which the bounds are derived, they are evaluated at $q^{2} \sim O\left(M e V^{2}\right)$. Thus, some extrapolation is necessary. It can be argued that, barring some bizarre behaviour, this is justified 12 .

An invisible decay mode with no new particles is the three body decay $\nu_{i} \rightarrow \nu_{j} \nu_{j} \bar{\nu}_{j}$. Even at the full strength of $\mathrm{Z}$ coupling, this yields a lifetime of $2.10^{34} \mathrm{~s}$, far too long to be of interest. There is an indirect bound from $\mathrm{Z}$ decays which is weaker but still yields $2.10^{30} \mathrm{~s}[13$.

Thus, the only decay modes which can have interestingly fast decays rates are two body modes such as $\nu_{i} \rightarrow \nu_{j}+x$ and $\nu_{i} \rightarrow \bar{\nu}_{j}+x$ where $x$ is a very light or massless particle, e.g. a Majoron.

The only possibility for fast invisible decays of neutrinos seems to lie with Majoron or Majoronlike models 9 . There are two classes of models; the I=1 Gelmini-Roncadelli[14 majoron and the $\mathrm{I}=0$ Chikasige-Mohapatra-Peccei [15] majoron. In general, one can choose the majoron to be a mixture of the two; furthermore the coupling can be to flavor as well as sterile neutrinos. The effective interaction is of the form:

$\bar{\nu}_{\beta}^{c}\left(a+b \gamma_{5}\right) \nu_{\alpha} J$

giving rise to decay:

$\nu_{\alpha} \rightarrow \bar{\nu}_{\beta}\left(\right.$ or $\left.\nu_{\beta}\right)+J$

where $J$ is a massless $J=0, L=2$ particle; $\nu_{\alpha}$ and $\nu_{\beta}$ are mass eigenstates which may be mixtures of flavor and sterile neutrinos. Models of this kind which can give rise to fast neutrino decays have been discussed [16]. These models are unconstrained by $\mu$ and $\tau$ decays which do not arise due to the $\Delta L=2$ nature of the coupling. The $\mathrm{I}=1$ coupling is constrained by the bound on the invisible $Z$ width; and requires that the Majoron be a mixture of $\mathrm{I}=1$ and $\mathrm{I}=017$. The couplings of $\nu_{\mu}$ and $\nu_{e}\left(g_{\mu}\right.$ and $\left.g_{e}\right)$ are constrained by the limits on multi-body $\pi, \mathrm{K}$ decays $\pi \rightarrow \mu \nu \nu \nu$ and $K \rightarrow \mu \nu \nu \nu$ and on $\mu-e$ university violation in $\pi$ and $K$ decays 18 , but not sufficiently strongly to rule out fast decays.

There are very interesting cosmological implications of such couplings. The details depend on the spectrum of neutrinos and the scalars in the model. For example, if all the neutrinos are heavier than the scalar; the relic neutrino density vanishes today, and the neutrino mass bounds from $\mathrm{CMB}$ and large scale structure are no longer operative, whereas future measurements in the laboratory might find a non-zero result for a neutrino mass [19]. If the scalars are heavier than the neutrinos, there are signatures such as shifts of the $n$th multipole peak (for large $n$ ) in the CMB 20]. There are other implications as well, such as the number of relativistic degrees of freedom(or effective number of neutrinos) being different at the $\mathrm{BBN}$ and the $\mathrm{CMB}$ eras. The additional degrees 
of freedom should be detectable in future CMB measurements.

Direct limits on such decay modes are also very weak. Current bounds on such decay modes are as follows. For the mass eigenstate $\nu_{1}$, the limit is about

$\tau_{1} \geq 10^{5} \mathrm{sec} / \mathrm{eV}$

based on observation of $\bar{\nu}_{e} s$ from SN1987A [21] (assuming CPT invariance). For $\nu_{2}$, strong limits can be deduced from the non-observation of solar anti-neutrinos in KamLAND 22 but only in the case when the coupling is to $\nu_{1}$. In the most general case, an analysis of solar neutrino data 23. leads to a bound given by:

$\tau_{2} \geq 10^{-4} \mathrm{sec} / \mathrm{eV}$

For $\nu_{3}$, in case of normal hierarchy, one can derive a bound from the atmospheric neutrino observations of upcoming neutrinos 24]:

$\tau_{3} \geq 10^{-10} \mathrm{sec} / \mathrm{eV}$

The strongest lifetime limit is thus too weak to eliminate the possibility of astrophysical neutrino decay by a factor about $10^{7} \times(L / 100 \mathrm{Mpc})$ $\times(10 \mathrm{TeV} / \mathrm{E})$. Some aspects of the decay of highenergy astrophysical neutrinos have been considered in the past. It has been noted that the disappearance of all states except $\nu_{1}$ would prepare a beam that could in principle be used to measure elements of the neutrino mixing matrix, namely the ratios $U_{e 1}^{2}: U_{\mu 1}^{2}: U_{\tau 1}^{2}[25$. The possibility of measuring neutrino lifetimes over long baselines was mentioned in Ref.[26], and some predictions for decay in four-neutrino models were given in Ref. [27]. We have shown that the particular values and small uncertainties on the neutrino mixing parameters allow for the first time very distinctive signatures of the effects of neutrino decay on the detected flavor ratios. The expected increase in neutrino lifetime sensitivity (and corresponding anomalous neutrino couplings) by several orders of magnitude makes for a very interesting test of physics beyond the Standard Model; a discovery would mean physics much more exotic than neutrino mass and mixing alone. As shown below, neutrino decay because of its unique signature cannot be mimicked by either different neutrino flavor ratios at the source or other nonstandard neutrino interactions.

A characteristic feature of decay is its strong energy dependence: $\exp (-L m / E \tau)$, where $\tau$ is the rest-frame lifetime. For simplicity, we will assume that decays are always complete, i.e., that these exponential factors vanish. The assumption of complete decay means we do not have to consider the distance and intensity distributions of sources. We assume an isotropic diffuse flux of high-energy astrophysical neutrinos, and can thus neglect the angular deflection of daughter neutrinos from the trajectories of their parents [28.

Disappearance only.- Consider the case of no detectable decay products, that is, the neutrinos simply disappear. This limit is interesting for decay to 'invisible' daughters, such as a sterile neutrino, and also for decay to active daughters if the source spectrum falls sufficiently steeply with energy. In the latter case, the flux of daughters of degraded energy will make a negligible contribution to the total flux at a given energy. Since coherence will be lost we have.

$$
\begin{aligned}
\phi_{\nu_{\alpha}}= & \sum_{i \beta} \phi_{\nu_{\beta}}^{\text {source }}(E)\left|U_{\beta i}\right|^{2}\left|U_{\alpha i}\right|^{2} e^{-L / \tau_{i}(E)} \\
& \stackrel{L \gg \tau_{i}}{\longrightarrow} \sum_{i(\text { stable }), \beta} \phi_{\nu_{\beta}}^{\text {source }}(E)\left|U_{\beta i}\right|^{2}\left|U_{\alpha i}\right|^{2},
\end{aligned}
$$

where the $\phi_{\nu_{\alpha}}$ are the fluxes of $\nu_{\alpha}, U_{\alpha i}$ are elements of the neutrino mixing matrix and $\tau$ are 
the neutrino lifetimes in the laboratory frame. Eq. (5) corresponds to the case where decay is complete by the time the neutrinos reach us, so only the stable states are included in the sum.

The simplest case (and the most generic expectation) is a normal hierarchy in which both $\nu_{3}$ and $\nu_{2}$ decay, leaving only the lightest stable eigenstate $\nu_{1}$. In this case the flavor ratio is $U_{e 1}^{2}: U_{\mu 1}^{2}: U_{\tau 1}^{2}[25]$. Thus if $U_{e 3}=0$

$\phi_{\nu e}: \phi_{\nu_{\mu}}: \phi_{\nu_{\tau}} \simeq 5: 1: 1$,

where we used the neutrino mixing parameters given above 8 . Note that this is an extreme deviation of the flavor ratio from that in the absence of decays. It is difficult to imagine other mechanisms that would lead to such a high ratio of $\nu_{e}$ to $\nu_{\mu}$. In the case of inverted hierarchy, $\nu_{3}$ is the lightest and hence stable state, and so 8

$\phi_{\nu_{e}}: \phi_{\nu_{\mu}}: \phi_{\nu_{\tau}}=U_{e 3}^{2}: U_{\mu 3}^{2}: U_{\tau 3}^{2}=0: 1: 1$.

If $U_{e 3}=0$ and $\theta_{a t m}=45^{\circ}$, each mass eigenstate has equal $\nu_{\mu}$ and $\nu_{\tau}$ components. Therefore, decay cannot break the equality between the $\phi_{\nu_{\mu}}$ and $\phi_{\nu_{\tau}}$ fluxes and thus the $\phi_{\nu_{e}}: \phi_{\nu_{\mu}}$ ratio contains all the useful information. The effect of a non-zero $U_{e 3}$ on the no-decay case of $1: 1: 1$ is negligible.

When $U_{e 3}$ is not zero, and the hierarchy is normal, it is possible to obtain information on the values of $U_{e 3}$ as well as the CPV phase $\delta[29$. The flavor ratio $e / \mu$ varies from 5 to 15 (as $U_{e 3}$ goes from 0 to 0.2 ) for $\cos \delta=+1$ but from 5 to 3 for $\cos \delta=-1$. The ratio $\tau / \mu$ varies from 1 to 5 $(\cos \delta=+1)$ or 1 to $0.2(\cos \delta=-1)$ for the same range of $U_{e 3}$.

If the decays are not complete and if the daughter does not carry the full energy of the parent neutrino; the resulting flavor mix is somewhat different but any case it is still quite distinct from the simple $1: 1: 1 \operatorname{mix} 8$.

Incidentally, neutrino decay also affects the signals for relic supernova $\bar{\nu}_{e} s$ and the sensitivity extends to $10^{10} \mathrm{sec} / \mathrm{eV}$. The main results can be summarized as follows 3031 . If we assume complete decay as before (for simplicity), then for normal hierarchy, the signal is enhanced by about a factor of 2 ; and for inverted hierarchy, the signal goes away.

\section{Magnetic Moments and Other Neutrino Properties}

If the path of neutrinos takes them thru regions with significant magnetic fields and the neutrino magnetic moments are large enough, the flavor mix can be affected 32 . The main effect of the passage thru magnetic field is the conversion of a given helicity into an equal mixture of both helicity states. This is also true in passage thru random magnetic fields 33 .

If the neutrino are Dirac particles, and all magnetic moments are comparable, then the effect of the spin-flip is to simply reduce the overall flux of all flavors by half, the other half becoming the sterile Dirac partners.

If the neutrinos are Majorana particles, the flavor composition remains $1: 1: 1$ when it starts from $1: 1: 1$, and the absolute flux remains unchanged.

What happens when large magnetic fields are present in or near the neutrino production region? In case of Dirac neutrinos, there is no difference and the outcoming flavor ratio remains $1: 1: 1$, with the absolute fluxes reduced by half. In case of Majorana neutrinos, since the initial flavor mix is no longer universal but is $\nu_{e}: \nu_{\mu}: \nu_{\tau} \approx 1$ : 2 : 0 , this is modified but it turns out that the final(post-oscillation) flavor mix is still $1: 1: 1$ ! 
As for mixing with sterile neutrinos, if the mixings are small, there are small deviations from the universality [7. A specific case of large mixing and very small $\delta m^{2}$ is discussed in the next section.

Other neutrino properties can also affect the neutrino flavor mix and modify it from the canonical $1: 1: 1$. If neutrinos have flavor(and equivalence principle) violating couplings to gravity(FVG), or Lorentz invariance violating(CPT violating or conserving) couplings; then there can be resonance effects which make for one way transitions(analogues of MSW transitions) e.g. $\nu_{\mu} \rightarrow$ $\nu_{\tau}$ but not vice versa 3435. In case of FVG for example, this can give rise to an anisotropic deviation of the $\nu_{\mu} / \nu_{\tau}$ ratio from 1 , becoming less than 1 for events coming from the direction towards the Great Attractor, while remaining 1 in other directions 34 .

Another possibility that can give rise to deviations of the flavor mix from the canonical $1: 1: 1$ is the idea of neutrinos of varying mass(MaVaNs). In this proposal [36, by having the dark energy and neutrinos(a sterile one to be specific) couple, and track each other; it is possible to relate the small scale $2 \times 10^{-3} \mathrm{eV}$ required for the dark energy to the small neutrino mass, and furthermore the neutrino mass depends inversely on neutrino density, and hence on the epoch. As a result, if this sterile neutrino mixes with a flavor neutrino, the mass difference varies along the path, with potential resonance enhancement of the transition probability into the sterile neutrino, and thus change the flavor mix 37]. For example, if only one resonance is crossed enroute, it can lead to a conversion of the lightest (mostly) flavor state into the (mostly) sterile state, thus changing the flavor mix to $1-U_{e 1}^{2}: 1-U_{\mu 1}^{2}: 1-U_{\tau 1}^{2} \approx 1 / 3:$ $1: 1$, in case of normal hierarchy and similarly $\approx 2: 1: 1$ in case of inverted hierarchy.

\section{Pseudo-Dirac Neutrinos with very small mass differences 38}

If each of the three neutrino mass eigenstates is actually a doublet with very small mass difference (smaller than $10^{-6} \mathrm{eV}$ ), then there are no current experiments that could have detected this. Such a possibility was raised long ago 39. It turns out that the only way to detect such small mass differences $\left(10^{-12} \mathrm{eV}^{2}>\delta m^{2}>10^{-18} \mathrm{eV}^{2}\right)$ is by measuring flavor mixes of the high energy neutrinos from cosmic sources. Fig. 1 shows that relic supernova neutrino signals and AGN neutrinos are sensitive to mass difference squared down to $10^{-20} \mathrm{eV}^{2}$.

Let $\left(\nu_{1}^{+}, \nu_{2}^{+}, \nu_{3}^{+} ; \nu_{1}^{-} \nu_{2}^{-}, \nu_{3}^{-}\right)$denote the six mass eigenstates where $\nu^{+}$and $\nu^{-}$are a nearly degenerate pair. A $6 \times 6$ mixing matrix rotates the mass basis into the flavor basis $\left(\nu_{e}, \nu_{\mu}, \nu_{\tau} ; \nu_{e} \nu_{\mu}, \nu_{\tau}\right)$. In general, for six Majorana neutrinos, there would be fifteen rotation angles and fifteen phases. However, for pseudo-Dirac neutrinos, Kobayashi and Lim [40] have given an elegant proof that the 6x6 matrix $V_{K L}$ takes the very simple form (to lowest order in $\delta m^{2} / \mathrm{m}^{2}$ :

$V_{K L}=\left(\begin{array}{cc}U & 0 \\ 0 & U_{R}\end{array}\right) \cdot\left(\begin{array}{cc}V_{1} & i V_{1} \\ V_{2} & -i V_{2}\end{array}\right)$,

where the $3 \times 3$ matrix $U$ is just the usual mixing matrix determined by the atmospheric and solar observations, the $3 \times 3$ matrix $U_{R}$ is an unknown unitary matrix and $V_{1}$ and $V_{2}$ are the diagonal matrices $V_{1}=\operatorname{diag}(1,1,1) / \sqrt{2}$, and $V_{2}=\operatorname{diag}\left(e^{-i \phi_{1}}, e^{-i \phi_{2}}, e^{-i \phi_{3}}\right) / \sqrt{2}$, with the $\phi_{i}$ being arbitrary phases.

As a result, the three active neutrino states are described in terms of the six mass eigenstates as:

$\nu_{\alpha L}=U_{\alpha j} \frac{1}{\sqrt{2}}\left(\nu_{j}^{+}+i \nu_{j}^{-}\right)$. 


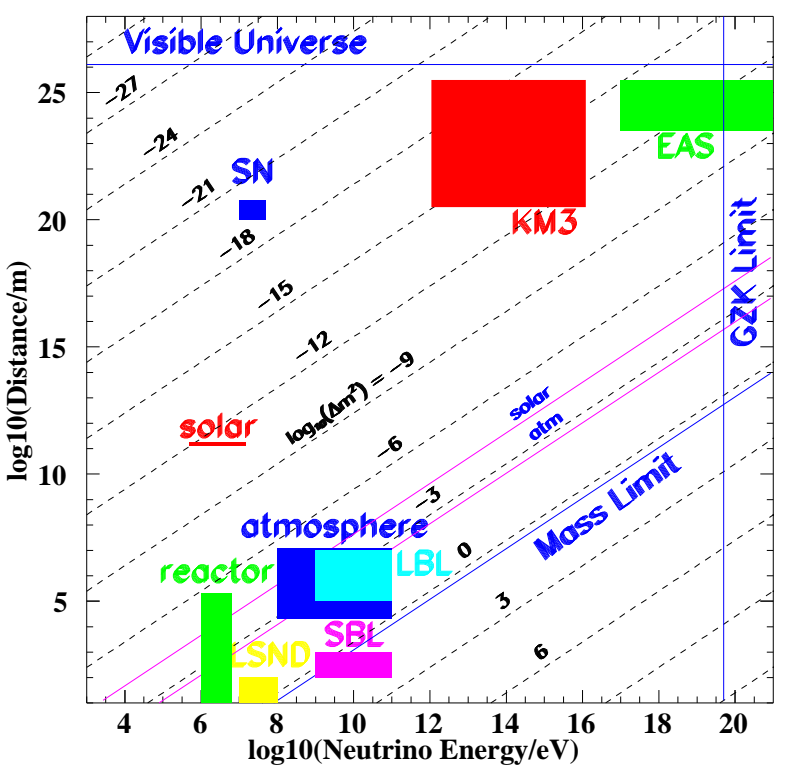

Figure 1. The ranges of distance and energy covered in various neutrino experiments. The diagonal lines indicate the mass-squared differences (in $\mathrm{eV}^{2}$ ) that can be probed with vacuum oscillations; at a given $L / E$, larger $\delta m^{2}$ values can be probed by averaged-out oscillations. We focus on a neutrino telescope of $1-\mathrm{km}$ scale (denoted "KM3"), or larger, if necessary. From Ref.[28].

The nontrivial matrices $U_{R}$ and $V_{2}$ are not accessible to active flavor measurements. The flavor conversion probability can thus be expressed as

$P_{\alpha \beta}=\frac{1}{4}\left|\sum_{j=1}^{3} U_{\alpha j}\left\{e^{i\left(m_{j}^{+}\right)^{2} L / 2 E}+e^{i\left(m_{j}^{-}\right)^{2} L / 2 E}\right\} U_{\beta j}^{*}\right|^{2}$

The flavor-conserving probability is also given by this formula, with $\beta=\alpha$. Hence, in the description of the three active neutrinos, the only new parameters are the three pseudo-Dirac mass differences, $\delta m_{j}^{2}=\left(m_{j}^{+}\right)^{2}-\left(m_{j}^{-}\right)^{2}$. In the limit that they are negligible, the oscillation formulas

\section{Table 1}

Flavor ratios $\nu_{e}: \nu_{\mu}$ for various scenarios. The numbers $j$ under the arrows denote the pseudoDirac splittings, $\delta m_{j}^{2}$, which become accessible as $L / E$ increases. Oscillation averaging is assumed after each transition $j$. We have used $\theta_{\text {atm }}=45^{\circ}$, $\theta_{\text {solar }}=30^{\circ}$, and $U_{e 3}=0$.

\begin{tabular}{ccccccc}
\hline \hline $1: 1$ & $\overrightarrow{3}$ & $4 / 3: 1$ & $\overrightarrow{2,3}$ & $14 / 9: 1$ & $\overrightarrow{1,2,3}$ & $1: 1$ \\
$1: 1$ & $\overrightarrow{1}$ & $2 / 3: 1$ & $\overrightarrow{1,2}$ & $2 / 3: 1$ & $\overrightarrow{1,2,3}$ & $1: 1$ \\
$1: 1$ & $\overrightarrow{2}$ & $14 / 13: 1$ & $\overrightarrow{2,3}$ & $14 / 9: 1$ & $\overrightarrow{\overrightarrow{1,2,3}}$ & $1: 1$ \\
$1: 1$ & $\overrightarrow{1}$ & $2 / 3: 1$ & $\overrightarrow{1,3}$ & $10 / 11: 1$ & $\overrightarrow{1,2,3}$ & $1: 1$ \\
$1: 1$ & $\overrightarrow{3}$ & $4 / 3: 1$ & $\overrightarrow{1,3}$ & $10 / 11: 1$ & $\overrightarrow{1,2,3}$ & $1: 1$ \\
$1: 1$ & $\overrightarrow{2}$ & $14 / 13: 1$ & $\overrightarrow{1,2}$ & $2 / 3: 1$ & $\overrightarrow{\overrightarrow{1,2,3}}$ & $1: 1$ \\
\hline \hline
\end{tabular}

reduce to the standard ones and there is no way to discern the pseudo-Dirac nature of the neutrinos.

$L / E$-Dependent Flavor Ratios.- Given the enormous pathlength between astrophysical neutrino sources and the Earth, the phases due to the relatively large solar and atmospheric masssquared differences will average out (or equivalently, decohere). The probability for a neutrino telescope to measure the flavor $\nu_{\beta}$ is then:

$$
P_{\beta}=\sum_{\alpha} w_{\alpha} \sum_{j=1}^{3}\left|U_{\alpha j}\right|^{2}\left|U_{\beta j}\right|^{2}\left[1-\sin ^{2}\left(\frac{\delta m_{j}^{2} L}{4 E}\right)\right]
$$

where $w_{\alpha}$ represents the fraction of the flavor $\alpha$ present initially. In the limit that $\delta m_{j}^{2} \rightarrow 0$, the expression above reproduces the standard form. The new oscillation terms are negligible until $E / L$ becomes as small as the tiny pseudo-Dirac masssquared splittings $\delta m_{j}^{2}$.

The flavors deviate from the democratic value of $\frac{1}{3}$ by

$$
\begin{aligned}
\delta P_{e} & =-\frac{1}{3}\left[\frac{3}{4} \chi_{1}+\frac{1}{4} \chi_{2}\right], \\
\delta P_{\mu}=\delta P_{\tau} & =-\frac{1}{3}\left[\frac{1}{8} \chi_{1}+\frac{3}{8} \chi_{2}+\frac{1}{2} \chi_{3}\right]
\end{aligned}
$$


where $\chi_{i}=\sin ^{2}\left(\delta m_{i}^{2} L / 4 E\right)$.

Table 1 shows how the $\nu_{e}: \nu_{\mu}$ ratio is altered if we cross the threshold for one, two, or all three of the pseudo-Dirac oscillations. The flavor ratios deviate from 1: 1 when one or two of the pseudo-Dirac oscillation modes is accessible. In the ultimate limit where $L / E$ is so large that all three oscillating factors have averaged to $\frac{1}{2}$, the flavor ratios return to $1: 1$, with only a net suppression of the measurable flux, by a factor of $1 / 2$.

\section{Cosmology with Neutrinos}

If the oscillation phases can indeed be measured for the very small mass differences by the deviations of the flavor mix from $1: 1: 1$ as discussed above, the following possibility is raised. It is a fascinating fact that non-averaged oscillation phases, $\delta \phi_{j}=\delta m_{j}^{2} t / 4 p$, and hence the factors $\chi_{j}$, are rich in cosmological information 2641]. Integrating the phase backwards in propagation time, with the momentum blue-shifted, one obtains

$$
\begin{aligned}
\delta \phi_{j} & =\int_{0}^{z_{e}} d z \frac{d t}{d z} \frac{\delta m_{j}^{2}}{4 p_{0}(1+z)} \\
& =\left(\frac{\delta m_{j}^{2} H_{0}^{-1}}{4 p_{0}}\right) I
\end{aligned}
$$

where I is given by

$I=\int_{1}^{1+z_{e}} \frac{d \omega}{\omega^{2}} \frac{1}{\sqrt{\omega^{3} \Omega_{m}+\left(1-\Omega_{m}\right)}}$,

$z_{e}$ is the red-shift of the emitting source, and $H_{0}^{-1}$ is the Hubble time, known to $10 \%$ [42]. This result holds for a flat universe, where $\Omega_{m}+\Omega_{\Lambda}=1$, with $\Omega_{m}$ and $\Omega_{\Lambda}$ the matter and vacuum energy densities in units of the critical density. The integral $I$ is the fraction of the Hubble time available for neutrino transit. For the presently preferred values $\Omega_{m}=0.3$ and $\Omega_{\Lambda}=0.7$, the asymptotic $\left(z_{e} \rightarrow \infty\right)$ value of the integral is 0.53 . This limit is approached rapidly: at $z_{e}=1(2)$ the integral is $77 \%(91 \%)$ saturated. For cosmologically distant $\left(z_{e}>1\right)$ sources such as gamma-ray bursts, non-averaged oscillation data would, in principle, allow one to deduce $\delta m^{2}$ to about $20 \%$, without even knowing the source red-shifts. Known values of $\Omega_{m}$ and $\Omega_{\Lambda}$ might allow one to infer the source redshifts $z_{e}$, or vice-versa.

This would be the first measurement of a cosmological parameter with particles other than photons. An advantage of measuring cosmological parameters with neutrinos is the fact that flavor mixing is a microscopic phenomena and hence presumably free of ambiguities such as source evolution or standard candle assumptions 26 43. Another method of measuring cosmological parameters with neutrinos is given in Ref. 444].

\section{Experimental Flavor Identification}

It is obvious from the above discussion that flavor identification is crucial for the purpose at hand. In a water cerenkov detector flavors can be identified as follows.

The $\nu_{\mu}$ flux can be measured by the $\mu^{\prime} s$ produced by the charged current interactions and the resulting $\mu$ tracks in the detector which are long at these energies. $\nu_{e}^{\prime} s$ produce showers by both $\mathrm{CC}$ and $\mathrm{NC}$ interactions. The total rate for showers includes those produced by $\mathrm{NC}$ interactions of $\nu_{\mu}^{\prime} s$ and $\nu_{\tau}^{\prime} s$ as well and those have to be (and can be) subtracted off to get the real flux of $\nu_{e}^{\prime} s$. However, this distinction between hadronic showers of neutral current events and the eletron-containing charged current events is rather difficult to make. Double-bang and lollipop events are signatures unique to tau neutrinos, made possible by the fact that tau leptons decay before they lose a 
significant fraction of their energy. Double-bang events consists of a hadronic shower initiated by a charged-current interaction of the $\nu_{\tau}$ followed by a second energetic shower (hadronic or electromagnetic) from the decay of the resulting tau lepton 4 4. Lollipop events consist of the second of the double-bang showers along with the reconstructed tau lepton track (the first bang may be detected or not). In principle, with a sufficient number of events, a fairly good estimate of the flavor ratio $\nu_{e}: \nu_{\mu}: \nu_{\tau}$ can be reconstructed, as has been discussed recently. Deviations of the flavor ratios from $1: 1: 1$ due to possible decays are so extreme that they should be readily identifiable 45]. Upcoming high energy neutrino telescopes, such as Icecube 46, will not have perfect ability to separately measure the neutrino flux in each flavor. However, the quantities we need are closely related to observables, in particular in the limit of $\nu_{\mu}-\nu_{\tau}$ symmetry $\left(\theta_{a t m}=45^{0}\right.$ and $U_{e 3}=0$ ), in which all mass eigenstates contain equal fractions of $\nu_{\mu}$ and $\nu_{\tau}$. In that limit, the fluxes for $\nu_{\mu}$ and $\nu_{\tau}$ are always in the ratio 1 : 1 , with or without decay. This is useful since the $\nu_{\tau}$ flux is the hardest to measure.

Even in the extreme case when one assumes that tau events are not identifiable, something about the flavor mix can be deduced. Let the only experimental information available be the number of muon tracks and the number of showers. The relative number of shower events to track events can be related to the most interesting quantity for testing decay scenarios, i.e., the $\nu_{e}$ to $\nu_{\mu}$ ratio. The precision of the upcoming experiments should be good enough to test the extreme flavor ratios produced by decays. If electromagnetic and hadronic showers can be separated, then the precision will be even better [45].
Comparing, for example, the standard flavor ratios of $1: 1: 1$ to the possible $5: 1: 1$ generated by decay, the more numerous electron neutrino flux will result in a substantial increase in the relative number of shower events.

The details of this observation depends on the range of muons generated in or around the detector and the ratio of charged to neutral current cross sections. The measurement will be limited by the energy resolution of the detector and the ability to reduce the atmospheric neutrino background. The atmospheric background drops rapidly with energy and should be negligibly small at and above the PeV scale.

\section{Discussion and Conclusions}

The flux ratios we discuss are energyindependent because we have assumed that the ratios at production are energy-independent, that all oscillations are averaged out, and that all possible decays are complete. In the standard scenario with only oscillations, the final flux ratios are $\phi_{\nu_{e}}: \phi_{\nu_{\mu}}: \phi_{\nu_{\tau}}=1: 1: 1$. In the cases with decay, we have found rather different possible flux ratios, for example $5: 1: 1$ in the normal hierarchy and $0: 1: 1$ in the inverted hierarchy. These deviations from $1: 1: 1$ are so extreme that they should be readily measurable.

If we are very fortunate [4], we may be able to observe a reasonable number of events from several sources (of known distance) and/or over a sufficient range in energy. Then the resulting dependence of the flux ratio $\left(\nu_{e} / \nu_{\mu}\right)$ on $\mathrm{L} / \mathrm{E}$ as it evolves from say 5 (or 0 ) to 1 , can be clear evidence of decay and further can pin down the actual lifetime instead of just placing a bound.

To summarize, we suggest that if future measurements of the flavor mix at earth of high en- 
ergy astrophysical neutrinos find it to be

$\phi_{\nu_{e}} / \phi_{\nu_{\mu}} / \phi_{\nu_{\tau}}=\alpha / 1 / 1$

then

(i) $\alpha \approx 1$ (the most boring case) confirms our knowledge of the MNSP 2] matrix and our prejudice about the production mechanism;

(ii) $\alpha \approx 1 / 2$ indicates that the source emits pure $\nu_{\mu}^{\prime} s$ and the mixing is conventional;

(iii) $\alpha \approx 3$ from a unique direction, e.g. the Cygnus region, would be evidence in favour of a pure $\bar{\nu}_{e}$ production as has been suggested recently [4];

(iv) $\alpha>1$ indicates that neutrinos are decaying with normal hierarchy; and

(v) $\alpha \ll 1$ would mean that neutrino decays are occuring with inverted hierarchy;

(vi) Values of $\alpha$ which cover a broader range (3 to 15) and deviation of the $\mu / \tau$ ratio from 1(between 0.2 to 5 ) can yield valuable information about $U_{e 3}$ and $\cos \delta$. Deviations of $\alpha$ which are less extreme(between 0.7 and 1.5) can also probe very small pseudo-Dirac $\delta m^{2}$ (smaller than $10^{-12} \mathrm{eV}^{2}$ ).

Incidentally, in the last three cases, the results have absolutely no dependence on the initial flavor mix, and so are completely free of any dependence on the production model. So either one learns about the production mechanism and the initial flavor mix, as in the first three cases, or one learns only about the neutrino properties, as in the last three cases. In any case, it should be evident that the construction of very large neutrino detectors is a "no lose" proposition.

\section{Acknowledgements}

This talk is based on published and ongoing work in collaboration with John Beacom, Nicole Bell, Dan Hooper, John Learned and Tom Weiler. I thank them for a most enjoyable collaboration; I also thank them for a careful and critical reading of the manuscript. I would like to acknowledge the splendid hospitality of the Fujihara foundation and Yoji Totsuka and Kenzo Nakamura in Tsukuba. This work was supported in part by U.S.D.O.E. under grant DE-FG03-94ER40833.

\section{REFERENCES}

1. S. Pakvasa, 9th International Symposium on Neutrino Telescopes, Venice, Italy, 69 Mar 2001, Venice 2001, Neutrino Telescopes, ed. M. Baldo-Ceolin, Vol. 2, p. 603; hep-ph/0105127

2. Z. Maki, M. Nakagawa and S. Sakata, Prog. Theoret. Phys. 28, 870 (1962); see also V. N. Gribov and B. M. Pontecorvo, Phys. Lett. B28, 493 (1969); the generalization to three flavors first appeared in B. W. Lee, S. Pakvasa, R. Shrock and H. Sugawara, Phys. Rev. Lett. 38, 937 (1977).

3. J. G. Learned and K. Mannheim, Ann. Rev. Nucl. Part. Sci. 50, 603 (2000), and references therein.

4. J. G. Learned and S. Pakvasa, Astropart. Phys. 3, 267 (1995); hep-ph/9405296

5. J. P. Rachen and P. Meszaros, Phys. Rev. D58, 123005 (1998); astro-ph/9802280

6. S. Pakvasa and J. Valle, Proc. Indian. Natl. Sci. Acad. 70A, 189 (2003); hep-ph/0301061

7. H. Athar, M. Jezabek and O. Yasuda, Phys. Rev. D62, 103007 (2000); hep-ph/0005104 L. Bento, P. Keranen and J. Maalampi, Phys. Lett. B476, 205 (2000); hep-ph/9912240 
8. J. F. Beacom, N. Bell, D. Hooper, S. Pakvasa and T.J. Weiler, Phys. Rev. Lett. 91, 181301 (2003); hep-ph/0211305

9. S. Pakvasa, Physics Potential and Development of Muon Colliders, Mu 99, San Francisco, AIP Conf. Proc. 542 (2000) 99, ed D. Cline, p. 99; hep-ph/0004077

10. J. F. Beacom and P. Vogel, Phys. Rev. Lett. 83, 5222 (1999); hep-ph/9970383

11. These are deduced 9 from the current bounds on the magnetic moments: C. Caso et al., Review of Particle Properties, Particle Data Group, Eur. Phys. J. C3, 1 (1998).

12. J-M Frere, R. B. Nezorov and M. Vysotsky, Phys. Lett. B394, 127 (1997); hep-ph/9608266

13. M. Bilenky and A. Santamaria, hep-ph/9908272 Phys. Lett. B301, 287 (1993).

14. G. Gelmini and M. Roncadelli, Phys. Lett. B99, 411 (1981).

15. Y. Chikasige, R. Mohapatra and R. Peccei, Phys. Rev. Lett. 45, 1926 (1980).

16. J. Valle, Phys. Lett., B131, 87 (1983); G. Gelmini and J. Valle, ibid. B142, 181 (1983); A. Joshipura and S. Rindani, Phys. Rev. D46, 3008 (1992); A. Acker, A. Joshipura and S. Pakvasa, Phys. Lett. B285, 371 (1992); A. Acker, S. Pakvasa and J. Pantaleone, Phys. Rev. D45, 1 (1992).

17. A. Choi and A. Santamaria, Phys.Lett. B267, 504 (1991).

18. V. Barger, W-Y. Keung and S. Pakvasa, Phys. Rev. D25, 907 (1982).

19. J.F. Beacom, N. Bell and S. Doddelson, hep-ph/0404585

20. Z. Chacko, L.J. Hall, T. Okui and S.J. Oliver, hep-ph/0312267
21. K. Hirata et al., Phys. Rev. Lett. 58, 1497 (1988); R.M. Bionta et al., ibid. 58, 1494 (1988).

22. K. Eguchi et al; Phys. Rev. Lett. 92, 071301 (2004); hep-ex/0310047

23. J. F. Beacom and N. Bell; Phys. Rev. D65, 113009 (2002); hep-ph/0204111 and references cited therein.

24. V. D. Barger, J. G. Learned, S. Pakvasa and T. J. Weiler, Phys. Rev. Lett. 82. 2640 (1999); hep-ph/9810121 Y. Ashie et al., hep-ex/0404034

25. S. Pakvasa, Lett. Nuov. Cimm. 31, 497 (1981); Y. Farzan and A. Smirnov, Phys. Rev. D65, 113001 (2002); hep-ph/0201105

26. T. J. Weiler, W. A. Simmons, S. Pakvasa and J. G. Learned; hep-ph/9411432

27. P. Keranen, J. Maalampi and J. T. Peltonieni, Phys. Lett. B461, 230 (1999); hep-ph/9901403

28. M. Lindner, T. Ohlsson and W. Winter, Nucl. Phys. B607, 326 (2001); ibid, B622, 429 (2002); astro-ph/0105309

29. J. F. Beacom, N. Bell, D. Hooper, S. Pakvasa and T. J. Weiler, Phys. Rev D69, 017303 (2004); hep-ph/0309267

30. G. L. Fogli, E. Lisi, A. Minzi and D. Montanino, hep-ph/0401227

31. S. Ando, Phys. Lett. B570, 11 (2003); hep-ph/0307169

32. K. Enqvist, P. Keranen and J. Maalampi, Phys. Lett.B438,295(1998); hep-ph/9806392

33. G. Domokos and S. Kovesi-Domokos, Phys. Lett B410, 57 (1997); hep-ph/9703265

34. H. Minakata and A. Yu. Smirnov, Phys. Rev. D54, 3698 (1996); hep-ph/9601311

35. V. D. Barger, S. Pakvasa, T. J. Weiler and K. Whisnant, Phys. Rev. Lett. 85, 5055 (2000); 
hep-ph/0005197.

36. R. Fardon, A. E. Nelson and N. Weiner, astro-ph/0309800

37. P. Q. Hung and H. Pas, astro-ph/0311131

38. J. F. Beacom, N. Bell, D. Hooper, J. G. Learned, S. Pakvasa and T. J. Weiler; Phys. Rev. Lett., 92 (2004); hep-ph/0307151 see also P. Keranen, J. Maalampi, M. Myyrylainen and J. Riittinen, Phys. Lett. B574, 162 (2003); hep-ph/0307041 for similar considerations.

39. L. Wolfenstein, Nucl. Phys. B186, 147 (1981); S. M. Bilenky and B. M. Pontecorvo, Sov. J. Nucl. Phys. 38, 248 (1983); S.T. Petcov, Phys. Lett. B110, 245 (1982).

40. M. Kobayashi and C. S. Lim, Phys. Rev. D64, 013003 (2001); hep-ph/0012266

41. D. J. Wagner and T. J. Weiler, Mod. Phys. Lett. A12, 2497 (1997).

42. W. L. Freedman et al. Astrophys. J. 553, 47 (2001).

43. L. Stodolsky, Phys. Lett. B473, 61 (2000); astro-ph/9911167

44. S. Choubey and S. F. King, Phys. Rev. D67, 073005 (2003), hep-ph/0207260

45. J. F. Beacom, N. Bell, D. Hooper, S. Pakvasa and T. J. Weiler; Phys. Rev. D68, 093005 (2003); hep-ph/0307025 F. Halzen and D. Hooper, Rept. Prog. Phys. 65, 1025 (2002); astro-ph/0204527.

46. A. Karle, Nucl. Phys. Proc. Supp., 118 (2003); astro-ph/0209556 A. Goldschmidt, Nucl. Phys. Proc. Suppl. 110, 516 (2002).

47. G. Barenboim and C. Quigg, Phys. Rev. D67, 073024 (2003); hep-ph/0301220

48. L. A. Anchordoqui, H. Goldberg, F. Halzen and T. J. Weiler, astro-ph/0311002. 